\title{
A nonlinear model for magnetoacoustic waves in dense dissipative plasmas with degenerate electrons
}

\author{
W. Masood ${ }^{1,2}$, R. Jahangir², B. Eliasson ${ }^{3}$, and M. Siddiq ${ }^{2}$ \\ ${ }^{1}$ COMSATS Institute of Information Technology, \\ Islamabad, Pakistan \\ ${ }^{2}$ National Centre for Physics (NCP), Shahdra Valley Road, \\ Islamabad, Pakistan \\ ${ }^{3}$ SUPA, Physics Department, University of Strathclyde, \\ Glasgow, United Kingdom \\ September 26, 2014
}

\begin{abstract}
The properties of nonlinear fast magnetoacoustic waves in dense dissipative plasmas with degenerate electrons are studied theoretically in the framework of the Zabolotskaya-Khokhlov (ZK) equation for small but finite amplitude excitations. Shock-like solutions of the ZK equation are obtained and are applied to parameters relevant to white dwarf stars.
\end{abstract}

\section{INTRODUCTION}

Plasmas with degenerate electrons having number densities comparable with solids and temperatures of several electron volts fall under the category of warm dense matter $[1,2]$ that exists in the core of giant planets $[3,4]$ and the crusts 
of old stars [5]. High-energy density physics [6] has gained interest due to its applications in astrophysical and cosmological environments [7, 8, 9, 10], as well as to inertial fusion science involving intense laser-solid density plasma interaction experiments $[11,12,13,14,15,16,17]$ and inertial confinement fusion [18].

The study of radiative blast waves in atomic cluster media using intense laser pulses has also been reported [19]. Atomic clusters have been shown to be very efficient absorbers of intense laser radiation. They can be used to create high energy density plasmas that drive strong shocks (>Mach 50) and radiative blast waves. Careful application of these equations and similarities allow experiments to be scaled to astrophysical phenomena that have spatial and temporal scales that are greater by as much as $15-20$ orders of magnitude. In this way, the radiative blast waves in the laboratory have been scaled those experienced in supernova remnants and the physics governing their dynamics investigated under controlled conditions.

An example of plasmas under extreme conditions is the compressed matter in white dwarf stars [20, 21, 22]. Pulsations observed in white dwarf stars are thought to be originating from gravity ( $g$-mode) waves in the inhomogeneous density of the star [23, 24], while compressional ( $p$-mode) waves are still to be observed. The lack of observations of $p$-mode waves may be due to that the $p$-modes act mainly in the vertical direction, where the vertical motions are limited by the huge gravity, leading to a very low amplitude of the oscillations below the detection limit [25]. Detectable, large amplitude acoustic waves could potentially be excited in extreme events, such as supernova explosions at the outer shells of the star or during collisions between the white dwarf with other astrophysical bodies. The self-steepening and shock formation in relativistically degenerate plasmas have been investigated theoretically [26], while linear and nonlinear magneto-acoustic waves in magnetized dense plasmas have been investigated for dissipative and non-dissipative cases [27, 28, 29, 30, 31].

In this paper, we derive the nonlinear Zabolotskaya-Khokhlov (ZK) equa- 
tion for magnetoacoustic waves in a dense dissipative plasma with degenerate electrons. The development of the ZK equation [32, 33, 34] and its variations in the 1960's to the 1980's was inspired by the interest in nonlinear acoustic beams together with the emerging developments in laser physics and the corresponding models of nonlinear optical beams. The equation sometimes goes under the name Khokhlov-Zabolotskaya equation [33, 34], and due to the contribution of Kuznetsov [35] to add a dissipative term to describe the propagation of nonlinear acoustic waves in a dissipative medium, it is sometimes denoted the Khokhlov-Zabolotskaya-Kuznetsov equation. This and other variations of the ZK equations have considerable interest in various fields of physics [34] and mathematics $[36,37]$. Since the ZK equation arises in many applications involving weak nonlinearity and quasi-plane waves, it is generally considered to be a canonical equation in the same way as the Laplace's equation is the canonical equation for linear elliptical systems. Even though equations related to the ZK equation are used to describe nonlinear magnetohydrodynamic waves in plasmas [38], the ZK equation itself is rarely mentioned in plasma physics. In Section 2, the model equations for the system are presented. The ZK equation is derived in Section 3, and shock-like solutions of the ZK equation are obtained by using Hirota's dependent variable transformation [39] and Clarkson-Kruskal approach $[40,41]$. Some applications of our results to magnetized white dwarf stars are also discussed. Finally, conclusions are drawn in Section 4.

\section{GOVERNING EQUATIONS}

We consider a dense magnetized plasma consisting of singly charged ions and inertialess Fermi-degenerate electrons. The magnetic field is directed along the $z$ direction, i.e. $\mathbf{B}=\left(0,0, B_{z}\right)$, and the spatial variations lie in the $x y$-plane. The normalized continuity and momentum equations describing the ion dynamics are

$$
\frac{\partial N_{i}}{\partial T}+\nabla \cdot\left(N_{i} \mathbf{V}_{i}\right)=0,
$$




$$
\frac{\partial \mathbf{V}_{i}}{\partial T}+\left(\mathbf{V}_{i} \cdot \nabla\right) \mathbf{V}_{i}=\mathbf{E}+\mathbf{V}_{i} \times \mathbf{B}+\mu_{1} \nabla^{2} \mathbf{V}_{i}+\left(\mu_{1}+\mu_{2}\right) \nabla\left(\nabla \cdot \mathbf{V}_{i}\right),
$$

where the ion thermal pressure has been neglected in comparison with the electron degeneracy pressure. Here the ion number density $N_{i}$ has been normalized by the equilibrium ion number density, $n_{i 0}$, the ion fluid velocity $\mathbf{V}_{i}$ has been normalized by the Alfvén speed, $V_{A}=B_{0} / \sqrt{\mu_{0} m_{i} n_{i o}}$, the magnetic field $\mathbf{B}$ has been normalized by the ambient magnetic field $B_{0}$, the electric field $\mathbf{E}=\left(E_{x}, E_{y}, 0\right)$ has been normalized by $V_{A} B_{0}, \mu_{1}=\mu_{1}^{\prime}\left(\omega_{c i} / V_{A}^{2}\right)$ is the normalized kinematic viscosity, and $\mu_{2}=\mu_{2}^{\prime}\left(\omega_{c i} / V_{A}^{2}\right)$ is the normalized second coefficient of viscosity. The kinematic viscosity $\mu_{1}^{\prime}$ can be estimated as $2.21 \times 10^{-15} T_{i}^{5 / 2} A_{i}^{1 / 2} /\left(Z^{4} \ln \Lambda\right)$ [42], where $A_{i}$ is the atomic weight of the ions, $Z$ is the ionic charge, $T_{i}$ is the ion temperature, and $\ln \Lambda$ is the logarithm of the Coulomb parameter. For a strongly magnetized charged fluid, the kinematic viscosity can be estimated as $2.68 \times 10^{-26} Z^{2} n_{i}^{2} A_{i}^{3 / 2} \ln \Lambda /\left(T_{i}^{1 / 2} B^{2}\right)$ [42]. More detailed descriptions of the viscosity in magnetized plasmas are given by Zhdanov [43] and references therein. The second coefficient of viscosity $\mu_{2}$ is put equal to $-(2 / 3) \mu_{1}[44,45]$. Here $\omega_{c i}=e B_{0} / m_{i}$ is the ion cyclotron frequency, $e$ is magnitude of the electron charge, $m_{i}$ is the ion mass, and $\mu_{0}$ is the magnetic permeability in vacuum. Time $T$ is normalized by $\omega_{c i}^{-1}$ and the space coordinates by the ion inertial length $V_{A} / \omega_{c i}$. The normalized continuity and momentum equations governing the dynamics of the degenerate inertialess electrons are given by

$$
\begin{gathered}
\frac{\partial N_{e}}{\partial T}+\nabla \cdot\left(N_{e} \mathbf{V}_{e}\right)=0, \\
0=-\left(\mathbf{E}+\mathbf{V}_{e} \times \mathbf{B}\right)-\beta \nabla N_{e}^{\frac{2}{3}} .
\end{gathered}
$$

where the electron number density $N_{e}$ has been normalized by the equilibrium electron number density $n_{e 0}=n_{i 0}$, the electron fluid velocity $\mathbf{V}_{e}$ has been normalized by $V_{A}, \beta=C_{s}^{2} / V_{A}^{2}, C_{s}=\sqrt{E_{F e} / m_{i}}$ is the ion acoustic speed, $E_{F e}=\hbar^{2}\left(3 \pi^{2} n_{e 0}\right)^{2 / 3} /\left(2 m_{e}\right)$ is the electron Fermi energy, $\hbar=h / 2 \pi$ is the reduced Planck's constant, $h$ is Planck's constant, and $m_{e}$ is the electron mass. 
The electric and magnetic fields are governed by Faraday's law

$$
\frac{\partial \mathbf{B}}{\partial T}=-\nabla \times \mathbf{E},
$$

and Ampère's law

$$
\nabla \times \mathbf{B}=N_{i} \mathbf{V}_{i}-N_{e} \mathbf{V}_{e}
$$

\section{LINEAR AND NONLINEAR ANALYSIS}

To study small but finite amplitude nonlinear magnetoacoustic waves, we stretch the independent variables as

$$
\begin{aligned}
\xi & =\epsilon^{1 / 2}\left(X-v_{0} T\right), \\
\eta & =\epsilon Y, \\
\tau & =\epsilon^{3 / 2} T,
\end{aligned}
$$

where $\epsilon$ is a small parameter measuring the weakness of the nonlinearity, and $v_{0}$ is the wave phase velocity (normalized by $V_{A}$ ). Assuming the kinematic viscosity to be small, we set $\mu=\epsilon^{1 / 2} \kappa$ where $\mu=2 \mu_{1}+\mu_{2}$. The perturbed quantities $N_{l}, V_{l x}, V_{l y}, B_{z}, E_{x}$ and $E_{y}$ are expanded in terms of power series of $\epsilon$ about their equilibrium values as:

$$
\begin{aligned}
N_{l} & =1+\epsilon N_{l}^{(1)}+\epsilon^{2} N_{l}^{(2)}+\epsilon^{3} N_{l}^{(3)}+\cdots, \\
V_{l x} & =0+\epsilon V_{l x}^{(1)}+\epsilon^{2} V_{l x}^{(2)}+\epsilon^{3} V_{l x}^{(3)}+\cdots, \\
V_{l y} & =0+\epsilon^{3 / 2} V_{l y}^{(1)}+\epsilon^{5 / 2} V_{l y}^{(2)}+\epsilon^{7 / 2} V_{l y}^{(3)}+\cdots, \\
B_{z} & =1+\epsilon B_{z}^{(1)}+\epsilon^{2} B_{z}^{(2)}+\epsilon^{3} B_{z}^{(3)}+\cdots, \\
E_{x} & =\epsilon^{3 / 2} E_{x}^{(1)}+\epsilon^{5 / 2} E_{x}^{(2)}+\epsilon^{7 / 2} E_{x}^{(3)}+\cdots, \\
E_{y} & =\epsilon E_{y}^{(1)}+\epsilon^{2} E_{y}^{(2)}+\epsilon^{3} E_{y}^{(3)}+\cdots
\end{aligned}
$$

where $l$ equals $e$ and $i$ for electrons and ions, respectively.

Using the stretched variables in Eqs. (1)-(6), we develop equations in differ- 
ent powers of $\epsilon$. For the lowest order of $\epsilon$, we obtain the following equations

$$
\begin{aligned}
N_{e}^{(1)} & =N_{i}^{(1)}=B_{z}^{(1)} \\
V_{e x}^{(1)} & =V_{i x}^{(1)}=E_{y}^{(1)}=v_{0} B_{z}^{(1)} \\
V_{i y}^{(1)} & =-E_{x}^{(1)}-v_{0}^{2} \frac{\partial B_{z}^{(1)}}{\partial \xi}, \\
V_{e y}^{(1)} & =-E_{x}^{(1)}-\frac{2}{3} \beta \frac{\partial B_{z}^{(1)}}{\partial \xi} .
\end{aligned}
$$

Solving the system (9) yields the dispersion relation

$$
v_{0}=\sqrt{1+\frac{2}{3} \beta}
$$

for linear magnetoacoustic waves.

Collecting the terms of order $\epsilon^{2}$, and using relations (9) and (10), we obtain

$$
\frac{\partial E_{x}^{(1)}}{\partial \xi}=-v_{0}^{2} \frac{\partial^{2} B_{z}^{(1)}}{\partial \xi^{2}}-v_{0} \frac{\partial B_{z}^{(1)}}{\partial \eta} .
$$

Finally, the set of equations of order $\epsilon^{5 / 2}$ (see the Appendix for details) leads to the Zabolotskaya-Khokhlov (ZK) equation

$$
\frac{\partial}{\partial \xi}\left(\frac{\partial B_{z}^{(1)}}{\partial \tau}+P B_{z}^{(1)} \frac{\partial B_{z}^{(1)}}{\partial \xi}-R \frac{\partial^{2} B_{z}^{(1)}}{\partial \xi^{2}}\right)+W \frac{\partial^{2} B_{z}^{(1)}}{\partial \eta^{2}}=0
$$

where

$$
\begin{aligned}
P & =\frac{3+(16 / 9) \beta}{2 \sqrt{1+(2 / 3) \beta}}, \\
R & =\frac{\kappa}{2} \\
W & =\frac{1}{2} \sqrt{1+\frac{2}{3} \beta},
\end{aligned}
$$

is the coefficient of nonlinearity, dissipation, and dispersion, respectively. We here briefly mention the assumptions used in the derivation of the ZK equation. The electron inertia and quantum diffraction effects have been ignored in the electron equation of motion, and since both these terms are dispersive in nature, the dispersion in the $\xi$-direction is absent in the ZK equation. However, the two-dimensionality of 
the problem leads to dispersion due to wave diffraction. Inclusion of the electron inertia would give the standard Kadomtsev-PetviashviliBurgers equation whereas excluding the second dimension $\eta$ (i.e. making the problem one-dimensional along $\xi$ ) and neglecting the electron inertia yields the standard Burgers equation. Application of the two-dimensional Burgers equation to weak shocks modified by the first-order Fermi acceleration of energetic electrons has been carried out by Zank and Webb [46]. Furthermore, in the current problem, if we ignore the viscosity, we get the dispersionless KadomtsevPetviashvili equation, whereas the one-dimensional case gives us the dissipation-free Burgers equation.

Special solutions of the ZK equation (12) can be found by applying numerous methods such as singular manifold method, Bäcklund transformation [47] etc. However, we here use Hirota's dependent variable method and ClarksonKruskal's approach $[39,40,41]$ that has been applied to many nonlinear evolution equations $[48,49,50,51]$. We assume that Eq. (12) possesses solutions of form

$$
B_{z}^{(1)}(\xi, \eta, \tau)=A \partial_{\xi}^{l} \partial_{\eta}^{m} \partial_{\tau}^{n} f[z(\xi, \eta, \tau)]+C_{1},
$$

where the constants $A$ and $C_{1}$ and the integers $l, m$, and $n$ are to be determined. The integers $l, m$, and $n$ are found by leading-order analysis [51, 52], which are $(1,0,0)$ for the ZK equation. Using symbolic computation, we compare the coefficient of highest power of $z_{\xi}$ and obtain an ordinary differential equation whose solution $f$ comes out to be

$$
f[z(\xi, \eta, \tau)]=-\frac{2}{A} \frac{R}{P} \log [z(\xi, \eta, \tau)] .
$$

Under the set of constraints obtained from the method and assuming $\xi$-linear form for $z(\xi, \eta, \tau)$, the solution of Eq. (12) is

$$
B_{z}^{(1)}(\xi, \eta, \tau)=-k \frac{R}{P} \tanh \left[\frac{1}{2}(k \xi+\alpha \eta+\omega \tau+\gamma)\right]-\frac{\omega}{k P}-\frac{\alpha^{2}}{k^{2}} \frac{W}{P} .
$$

The obtained solution describes a two-dimensional shock structure, where $\gamma, k$, $\alpha$, and $\omega$ are constants. By choosing a coordinate system such that $\gamma=0$, and 
defining the shock amplitude as $B_{z 0}=2 k R / P$ and $\omega=-\alpha^{2} W / k-k^{2} R$, gives a solution that has amplitude $B_{z}^{(1)}=B_{z 0}$ at $k \xi+\alpha \eta+\omega \tau \rightarrow-\infty$ and $B_{z}^{(1)}=0$ at $k \xi+\alpha \eta+\omega \tau \rightarrow+\infty$. Choosing $\alpha=k \tan (\theta)$ where $\theta$ is the angle between the shock normal and the $\xi$-axis, the solution can be written

$$
B_{z}^{(1)}(\xi, \eta, \tau)=\frac{B_{z 0}}{2}\left(1-\tanh \left\{\Delta\left[(\xi+\eta \tan \theta)-V_{0} \tau\right]\right\}\right),
$$

where $\Delta=B_{z 0} P /(4 R)$ is the shock width and $V_{0}=B_{z 0} P / 2+W \tan ^{2} \theta$ is the modification of the shock speed due to nonlinear and geometric effects. The shock width varies due to an inter-play between nonlinearity and dissipation while the shock speed increases due to nonlinear effects and diffraction.

A wide range of values exist for the number densities and magnetic fields $[53,54,55]$ in white dwarf stars. Typical values (for non-relativistic densities) are $n_{i}=10^{32}-10^{35} \mathrm{~m}^{-3}$ and $B_{0}=10^{2}-10^{5} \mathrm{~T}$. The values $T_{i}=3.5 \times 10^{4} \mathrm{~K}$, $n_{i 0}=10^{32} \mathrm{~m}^{-3}$ and $B_{0}=10^{5} \mathrm{~T}$ give $\beta=0.06, \kappa=0.03, R=0.015, P=1.52$ and $W=0.51$.

\section{CONCLUSION}

In this paper, we have presented a theory for electromagnetic shock structures in dense dissipative plasmas consisting of non-degenerate ions and degenerate inertialess electrons, and have derived the Zabolotskaya-Khokhlov (ZK) equation. Even though equations related to the ZK equation are used to model nonlinear magnetohydrodynamic waves in plasmas [38], the ZK equation itself is rarely mentioned in plasma physics. We have also discussed the assumptions under which the ZK equation can be obtained in plasmas and the physical meaning has also been discussed. Shock solution of the ZK equation has been obtained by combining Hirota's and Clarkson-Kruskal approaches. It has been pointed out that limiting cases of the ZK equation yield the dispersionless Kadomtsev-Petviashvili equation and the one-dimensional Burgers equation while the inclusion of some dispersive effects would yield the Kadomtsev-Petviashvili-Burgers equation. It should be men- 
tioned that although the results presented here are for degenerate plasmas such as in the outer shells of white dwarf stars, the theoretical construct is general and could be applied to classical plasmas as well.

\section{Appendix}

Using the perturbation scheme, we collect to the lowest order $\epsilon^{1}$ the $y$-components of the ion and electron momentum equations and $x$-component of Ampère's law, to obtain

$$
\begin{aligned}
& E_{y}^{(1)}-V_{i x}^{(1)}=0 \\
& V_{e x}^{(1)}-E_{y}^{(1)}=0 \\
& V_{e x}^{(1)}-V_{i x}^{(1)}=0 .
\end{aligned}
$$

To the next higher order $\epsilon^{\frac{3}{2}}$, the ion and electron continuity equations and the $x$-components of the ion and electron momentum equations yield

$$
\begin{aligned}
-v_{0} \frac{\partial N_{i}^{(1)}}{\partial \xi}+\frac{\partial V_{i x}^{(1)}}{\partial \xi} & =0 \\
-v_{0} \frac{\partial N_{e}^{(1)}}{\partial \xi}+\frac{\partial V_{e x}^{(1)}}{\partial \xi} & =0 \\
-v_{0} \frac{\partial V_{i x}^{(1)}}{\partial \xi}-E_{x}^{(1)}-V_{i y}^{(1)} & =0 \\
-\frac{2}{3} \beta \frac{\partial N_{e}^{(1)}}{\partial \xi}-E_{x}^{(1)}-V_{e y}^{(1)} & =0
\end{aligned}
$$

while Faraday's law and the $y$-component of Ampère's law give

$$
\begin{gathered}
-v_{0} \frac{\partial B_{z}^{(1)}}{\partial \xi}+\frac{\partial E_{y}^{(1)}}{\partial \xi}=0, \\
\frac{\partial B_{z}^{(1)}}{\partial \xi}-V_{e y}^{(1)}+V_{i y}^{(1)}=0 .
\end{gathered}
$$

These equations are used in Eqs. (9) and (10), where the $\epsilon^{2}$-order terms of the $y$-component of the ion and electron momentum equations and $x$-component of 
Ampère's law give

$$
\begin{aligned}
E_{y}^{(2)}-V_{i x}^{(2)}-V_{i x}^{(1)} B_{z}^{(1)}+v_{0} \frac{\partial V_{i y}^{(1)}}{\partial \xi} & =0, \\
E_{y}^{(2)}-V_{e x}^{(2)}-V_{e x}^{(1)} B_{z}^{(1)}+\frac{2}{3} \beta \frac{\partial N_{e}^{(1)}}{\partial \eta} & =0, \\
\frac{\partial B_{z}^{(1)}}{\partial \eta}+V_{e x}^{(2)}-V_{i x}^{(2)}+N_{e}^{(1)} V_{e x}^{(1)}-N_{i}^{(1)} V_{i x}^{(1)} & =0 .
\end{aligned}
$$

The above set of equations is used to obtain the relation between $E_{x}^{(1)}$ and $B_{z}^{(1)}$. The next order $\epsilon^{5 / 2}$ of the ion and electron continuity equations and the $x$-components of the ion and electron momentum equations are

$$
\begin{aligned}
-v_{0} \frac{\partial N_{i}^{(2)}}{\partial \xi}+\frac{\partial V_{i x}^{(2)}}{\partial \xi} & =-\frac{\partial N_{i}^{(1)}}{\partial \tau}-\frac{\partial\left(N_{i}^{(1)} V_{i x}^{(1)}\right)}{\partial \xi}-\frac{\partial V_{i y}^{(1)}}{\partial \eta}, \\
-v_{0} \frac{\partial N_{e}^{(2)}}{\partial \xi}+\frac{\partial V_{e x}^{(2)}}{\partial \xi} & =-\frac{\partial N_{e}^{(1)}}{\partial \tau}-\frac{\partial\left(N_{e}^{(1)} V_{e x}^{(1)}\right)}{\partial \xi}-\frac{\partial V_{e y}^{(1)}}{\partial \eta}, \\
-v_{0} \frac{\partial V_{i x}^{(2)}}{\partial \xi}-E_{x}^{(2)}-V_{i y}^{(2)} & =-\frac{\partial V_{i x}^{(1)}}{\partial \tau}-V_{i x}^{(1)} \frac{\partial V_{i x}^{(1)}}{\partial \xi}+V_{i y}^{(1)} B_{z}^{(1)}+\kappa \frac{\partial^{2} V_{i x}^{(1)}}{\partial \xi^{2}}, \\
\frac{2}{3} \beta \frac{\partial N_{e}^{(2)}}{\partial \xi}+E_{x}^{(2)}+V_{e y}^{(2)} & =-V_{e y}^{(1)} B_{z}^{(1)}+\frac{2}{9} \beta N_{e}^{(1)} \frac{\partial N_{e}^{(1)}}{\partial \xi},
\end{aligned}
$$

while Faraday's law and the $y$-component of Ampere's law yield

$$
\begin{aligned}
-v_{0} \frac{\partial B_{z}^{(2)}}{\partial \xi}+\frac{\partial E_{y}^{(2)}}{\partial \xi}+\frac{\partial B_{z}^{(1)}}{\partial \tau}-\frac{\partial E_{x}^{(1)}}{\partial \eta} & =0, \\
-\frac{\partial B_{z}^{(2)}}{\partial \xi}+V_{e y}^{(2)}-V_{i y}^{(2)}+N_{e}^{(1)} V_{e y}^{(1)}-N_{i}^{(1)} V_{i y}^{(1)} & =0 .
\end{aligned}
$$

The coupled equations (21)-(23) along with Eqs. (10) and (11) are solved to eliminate $N_{i}^{(2)}, N_{e}^{(2)}, V_{i x}^{(2)}, V_{e x}^{(2)}, V_{i y}^{(2)}, V_{e y}^{(2)}, E_{y}^{(2)}$ and $E_{x}^{(2)}$ to obtain the ZK equation (12).

\section{References}

[1] S. Ichimaru, Rev. Mod. Phys. 54, 1017 (1982).

[2] V. E. Fortov, Phys. Usp. 52, 615 (2009).

[3] H. M. Horn, Science. 252, 384 (1991). 
[4] G. Chabrier, Plasma Phys. Controlled Fusion 51, 124014 (2009).

[5] T. Guillot, Science 286, 72 (1999).

[6] R. P. Drake, Phys. Plasmas 16, 055501 (2009).

[7] D. Lai, Rev. Mod. Phys. 73, 629 (2001).

[8] M. Opher, L. O. Silva, D. E. Dauger, V. K. Decyk, and J. M. Dawson, Phys. Plasmas 8, 2454 (2001).

[9] O. G. Benvenuto, and M. A. De Vito, Mon. Not. R. Astron. Soc. 362, 891 (2005).

[10] A. K. Harding, and D. Lai, Rep. Prog. Phys. 69, 2631 (2006).

[11] S. X. Hu, and C. H. Keitel, Phys. Rev. Lett. 83, 4709 (1999).

[12] A. V. Andreev, JETP Lett. 72, 238 (2000).

[13] J. T. Mendonça, Theory of Photon Acceleration (Institute of Physics, Bristol) (2001).

[14] S. H. Glenzer, O. L. Landen, P. Neumayer, R. W. Lee, K. Widmann, S. W. Pollaine, R. J. Wallace, G. Gregori, A. Höll, T. Bornath, R. Thiele, V. Schwarz, W.-D. Kraeft, and R. Redmer, Phys. Rev. Lett. 98, 065002 (2007).

[15] Andrea L. Kritcher, Paul Neumayer, John Castor, Tilo Döppner, Roger W. Falcone, Otto L. Landen, Hae Ja Lee, Richard W. Lee, Edward C. Morse, Andrew Ng, Steve Pollaine, Dwight Price, and Siegfried H. Glenzer, Science 322, 69 (2008).

[16] P. Neumayer, C. Fortmann, T. Döppner, P. Davis, R. W. Falcone, A. L. Kritcher, O. L. Landen, H. J. Lee, R. W. Lee, C. Niemann, S. Le Pape, and S. H. Glenzer, Phys. Rev. Lett. 105, 075003 (2010). 
[17] D. H. Froula, S. H. Glenzer, N.C. Luhmann, Jr., and J. Sheffield, Plasma Scattering of Electromagnetic Radiation: Theory and Measurement Techniques (Academic Press, New York), 2nd ed. (2011).

[18] H. Azechi and the FIREX project, Plasma Phys. Controlled Fusion 48, B267 (2006).

[19] Bull. Am. Phys. Soc. 53, 221 (2008).

[20] S. Balberg and S. L. Shapiro, The Properties of Condensed Matter in White Dwarfs and Neutron Stars, in M. Levy, H. Bass, R. Stern, and R. Paspet (Eds.) Handbook of Elastic Properties of Solids, Liquids, and Gases (Academic Press, U. K., 2000) Volume IV, Chapter 7; arXiv:astro$\mathrm{ph} / 0004317 \mathrm{v} 2$.

[21] G. Fontaine and F. Wesemae, White Dwarfs, in P. Murdin (Ed.) Encyclopedia of Astronomy and Astrophysics (Nature Publishing Group, New York and Tokyo, 2001), p. 3525.

[22] S. I. Shapiro and S. A. Teukolsky, Black Holes, White Dwarfs, and Neutron Stars: The Physics of Compact Objects (Wiley, new York, 1983).

[23] D. E. Winget and S. O. Kepler, Annu. Rev. Astron. Astrophys. 46, 157 (2008).

[24] G. Fontaine and P. Brassard, Publ. Astron. Soc. Pac. 120, 1043 (2008).

[25] R. Silvotti, G. Fontaine, Pavlov M. et al Astron. Astrophys. 525, A64 (2011).

[26] B. Eliasson and P. K. Shukla, EPL 97, 15001 (2012).

[27] G. Brodin and M. Marklund, New J. Phys. 9, 277 (2007).

[28] M. Marklund and G. Brodin, Phys. Rev. Lett. 98, 025001 (2007).

[29] W. Masood and A. Mushtaq, Phys. Lett. A. 372, 4283 (2008). 
[30] W. Masood, Phys. Plasmas. 16, 042314 (1-8) (2009).

[31] W. Masood, B. Eliasson, and P. K. Shukla, Phys. Rev. E. 81, 066401 (2010).

[32] E. A. Zabolotskaya and R. V. Khokhlov, Sov. Phys. Acoustics. 15, 35 (1969).

[33] O. V. Rudenko, V. A. Khokhlova, and M. F. Hamilton, J. Acoust. Soc. Am. 126, 2200 (2009).

[34] O. V. Rudenko, Acoustical Physics 56(4) 457 (2010).

[35] V. P. Kuznetsov, Akust. Zh. 16, 548 (1970) [Sov. Phys. Acoust. 16, 467 (1970)].

[36] H. Segur, Physica D 18, 1 (1986).

[37] G. M. Webb and G. P. Zank, J. Phys. A: Math. Gen. 23, 5465 (1990).

[38] V. Yu. Belashov and S. V. Vladimirov, Solitary Waves in Dispersive Complex Media, Springer Series in Solid-State Sciences (Springer-Verlag, Berlin and Heidelberg 2005).

[39] R. Hirota, The Direct Method in Soliton Theory, Cambridge University Press, 2004.

[40] P. A. Clarkson and M. D. Kruskal, J. Math. Phys 30, 2201 (1989).

[41] M. C. Nucci and P. A. Clarkson, Phys Lett A 16449 (1992).

[42] Jr. Spitzer, Physics of Fully Ionized Gases (John Wiley \& Sons, New York, (1962), pp. 146.

[43] V. M. Zhdanov and A. A. Stepanenko, Plasma Physics Reports, 39, 976 (2013).

[44] L. D. Landau and E. M. Lifshitz, Fluid Mechanics (Pergamon, Oxford,1959). 
[45] D. J. Tritton, Physical Fluid Dynamics (Clarendon, Oxford, 1988).

[46] G. P. Zank and G. W. Webb, J Plasma. Phys 44, 91 (1990).

[47] M.R. Miura, Backlund transformation (Springer-Verlag, Berlin,1978).

[48] Sirendaoreji, J. Phys. A: Math. Gen. 32, 6897 (1999).

[49] J. Hietarinta, J. Phys. A: Math. Gen. 33, 5157 (2000).

[50] Y. T. Gao and B. Tian, Comput. Math. Appl. 30, 97 (1995).

[51] B. Tian and Y. T. Gao, Phys. A: Math. Gen. 29, 2895 (1996).

[52] J. Weiss, M. Tabor and G. Carnevale, J. Math. Phys. 24, 522 (1983).

[53] H. M. Vann Horn, Phys. Today 32, 1, 23 (1979).

[54] T. Padmanabhan, Theoretical Astrophysics, Volume II: Stars and Stellar Systems Cambridge University Press, London, (2001).

[55] D. Koester and G. Chanmugam, Rep. Prog. Phys. 53, 837 (1990). 